\title{
Adenovirus Pneumonia Presenting with Nodular Shadows on Chest X-ray in Two Unrelated Allogeneic Bone Marrow Transplant Recipients
}

\author{
Kanako Mochizuki ${ }^{1}$, Yukio Kondo ${ }^{1}$, Kohei Hosokawa ${ }^{1}$, Kinya Ohata ${ }^{1}$, Hirohito Yamazaki ${ }^{1}$, \\ Akiyoshi Takami ${ }^{1}$, Motoko Sasaki ${ }^{2}$, Yasunori Sato ${ }^{2}$, Yasuni Nakanuma ${ }^{2}$ and Shinji Nakao ${ }^{1}$
}

\begin{abstract}
Adenoviruses are increasingly recognized as important pathogens following allogeneic stem cell transplantation. We herein report two cases of disseminated adenovirus infection that presented with nodular shadows on chest X-ray after allogeneic bone marrow transplantation from unrelated donors. Both patients died of respiratory failure. Autopsies revealed adenovirus infection of multiple organs. Adenovirus infection should be suspected when nodular lung lesions of unknown origin appear in allogeneic stem cell transplant recipients.
\end{abstract}

Key words: adenovirus, pneumonia, nodular shadows, disseminated infection, allogeneic stem cell transplantation

(Intern Med 53: 499-503, 2014)

(DOI: 10.2169/internalmedicine.53.1192)

\section{Introduction}

Adenovirus infections exhibit a worldwide distribution and occur throughout the year. Although most cases are selflimited, infections in immunocompromised hosts can be fatal. The clinical manifestations of adenovirus infection in transplant recipients range from asymptomatic excretion to disseminated infection with multiorgan failure and death (1). Cidofovir is currently the most effective agent in such cases, although it often demonstrates limited efficacy in severely immunocompromised patients (2).

We herein report two cases of disseminated adenoviral infection in which the patients displayed nodular shadows on chest X-rays and were diagnosed at autopsy. We also discuss the relevance of previously reported risk factors for adenovirus infection in these cases.

\section{Case Reports}

\section{Case 1}

A 50-year-old man was diagnosed with $\operatorname{IgA}-\lambda$ multiple myeloma in March 2006. He achieved a partial response to the VAD (vincristine, doxorubicin and dexamethasone) regimen and received an autologous peripheral blood stem cell transplant following high-dose melphalan therapy. He developed hepatomegaly and systemic lymphadenopathy in June 2007 and was diagnosed with diffuse large B-cell lymphoma due to the transformation of multiple myeloma. Following the administration of chemotherapy with the R-ESHAP (rituximab, etoposide, methylprednisolone, high-dose cytarabine and cisplatin) and R-HyperCVAD (rituximab, hyperfractionated cyclophosphamide, vincristine, doxorubicin and dexamethasone) regimens, he received an allogeneic bone marrow transplant (BMT) from an HLA-matched unrelated donor with a reduced-intensity conditioning regimen in February 2008. He subsequently developed abdominal pain and macroscopic hematuria on day 20 post-transplantation. Com-

\footnotetext{
${ }^{1}$ Department of Cellular Transplantation Biology, Division of Cancer Medicine, Kanazawa University Graduate School of Medical Science, Japan and ${ }^{2}$ Department of Human Pathology, Kanazawa University Graduate School of Medical Science, Japan

Received for publication June 18, 2013; Accepted for publication September 6, 2013

Correspondence to Dr. Kanako Mochizuki, kanamoc@gmail.com
} 
A

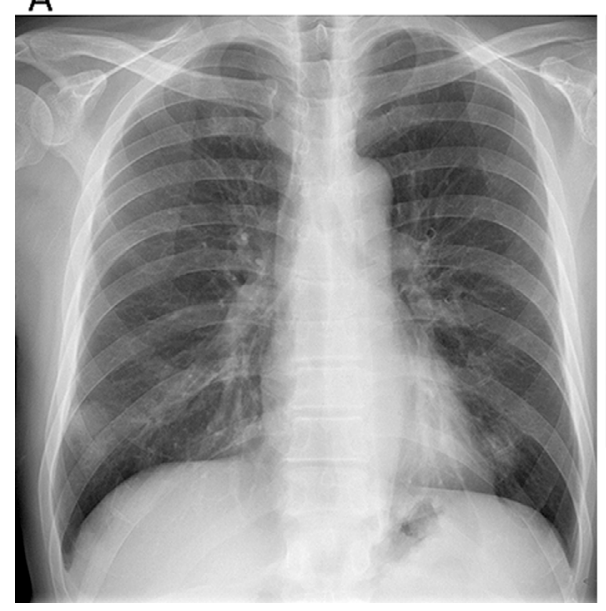

B

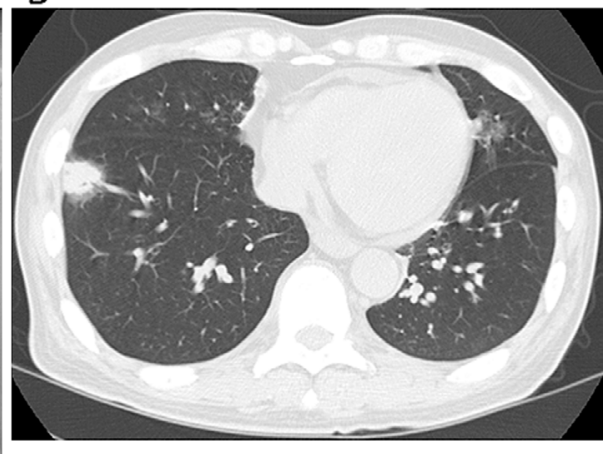

Figure 1. Chest radiograph and CT images in Case 1. A: A chest radiograph showing bilateral patchy areas. B: A CT image showing a nodule surrounded by ground-glass attenuation in the right lung and patchy ground-glass attenuation in the left lung.
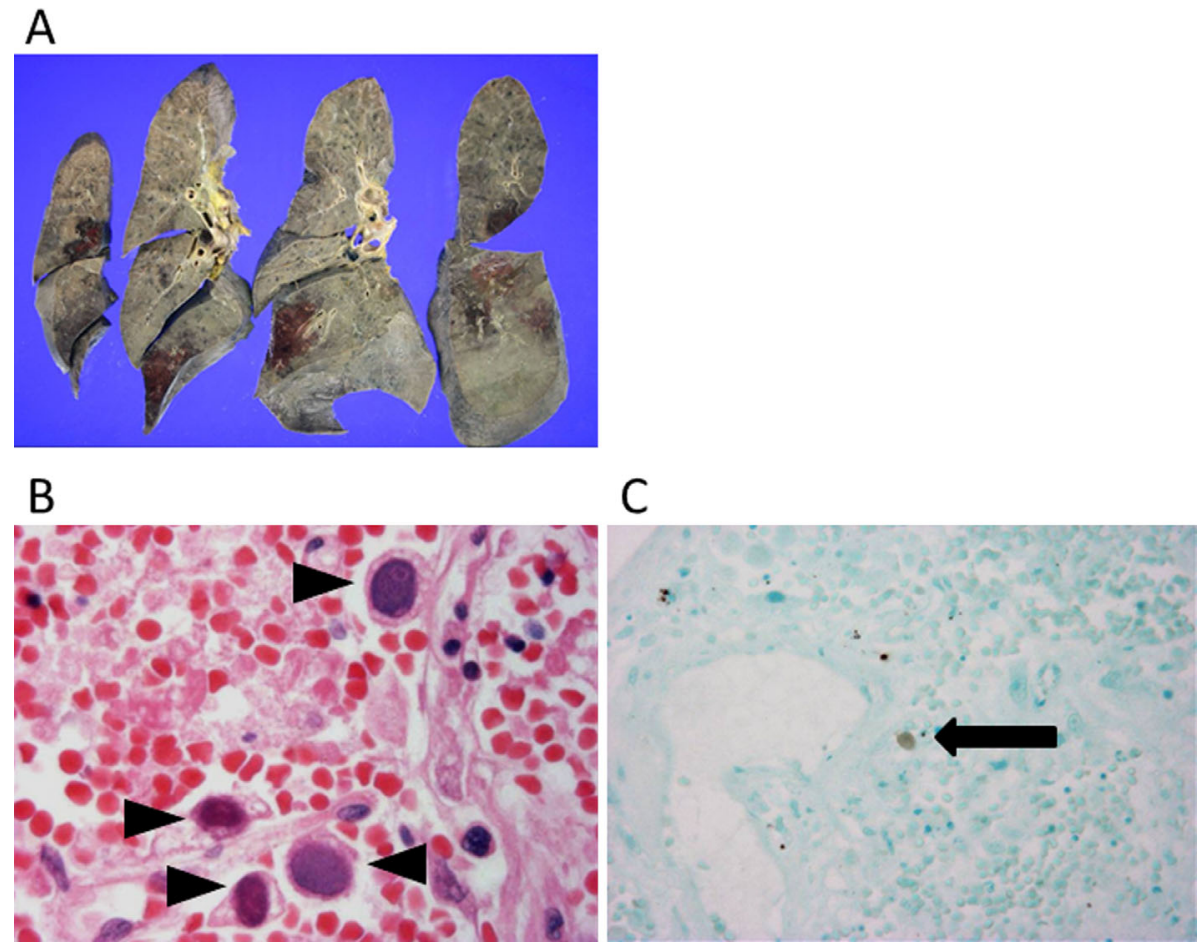

Figure 2. Autopsy findings in Case 1. A: Macroscopic findings showing multiple hemorrhagic areas with necrosis in both lungs. B: A Hematoxylin and Eosin stained lung specimen showing virus-infected lung alveolar epithelial cells containing intranuclear inclusion bodies $(\times 1,000)$. Arrowheads: smudge cells. C: Immunohistochemical staining for adenovirus $(\times 400)$.

puted tomography (CT) images showed bilateral hydronephrosis and hydroureter. Adenovirus was detected in the patient's urine, but not in his peripheral blood, on PCR. He was diagnosed with hemorrhagic cystitis caused by adenovirus. Continuous bladder irrigation gradually relieved his abdominal pain. However, he began to complain of dyspnea on day 38. CT images revealed lung nodules surrounded by ground-glass attenuation Fig. 1A, B). Despite treatment with various antibiotics, antifungal agents and ganciclovir, his respiratory status deteriorated. He died of respiratory failure on day 58 post-transplantation. An autopsy was performed 8.5 hours after his death, which revealed multiple hemorrhagic areas with necrosis in both lungs (Fig. 2A). A microscopic examination demonstrated virus-infected lung alveolar epithelial cells containing intranuclear inclusion bodies (Fig. 2B) that were positive for adenovirus immunostaining using anti-adenovirus antibodies specific to a hexon polypeptide (Fig. 2C) and negative for cytomegalovirus and herpesvirus. Virus-infected cells were also found in the kidneys, lower esophagus and jejunum. Therefore, a pathologi- 
A

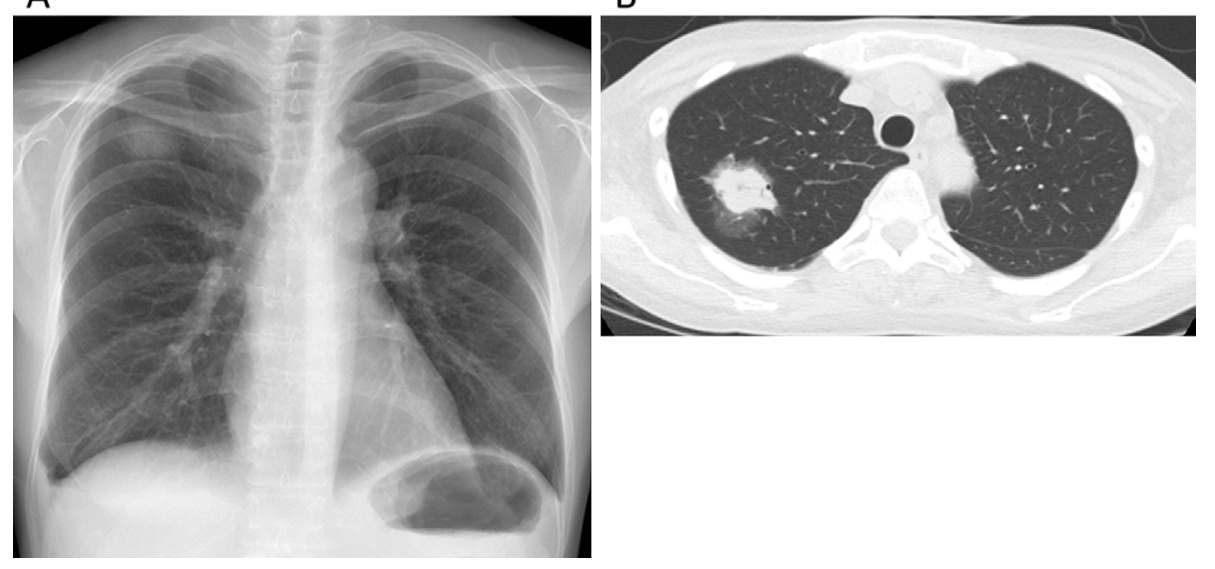

Figure 3. Chest radiograph and CT images in Case 2. A: A chest radiograph showing a nodule in the right lung. B: A CT image showing a nodule surrounded by a halo of ground-glass attenuation.

cal diagnosis of disseminated adenovirus infection was made.

\section{Case 2}

In October 2008, a 41-year-old woman received an allogeneic BMT for the treatment of acute myelogenous leukemia. Despite achieving complete donor chimerism, the patient's hematological recovery was incomplete, and she required regular transfusions of red blood cells and platelets. Tacrolimus was discontinued on day 199, as there were no signs of graft-versus-host disease (GVHD). Approximately 10 months post-transplantation, she developed erythema, liver dysfunction with an elevated serum bilirubin level and sicca symptoms. She was diagnosed with extensive chronic GVHD based on the findings of skin and liver biopsies, and treatment with $1 \mathrm{mg} / \mathrm{kg}$ of oral prednisolone was initiated. Cyclosporine was added 70 days after the start of treatment with prednisolone alone. Although the patient's erythema and sicca symptoms improved, the liver dysfunction further deteriorated. A chest X-ray performed on day 427 showed a nodule in the right upper lung (Fig. 3A). Thin-section CT images revealed a 30-mm nodule surrounded by a halo of ground-glass attenuation in the upper lobe of the right lung (Fig. 3B). Despite intensive treatment with antifungal agents, antibiotics and ganciclovir, the patient's pneumonia rapidly progressed. Peritonitis occurred on day 430, and she died of multiorgan failure on day 435.

An autopsy was performed two hours after her death, which revealed a $40 \times 30-\mathrm{mm}$ necrotic lesion surrounded by a hemorrhagic area in the right upper lobe with scattered foci of hemorrhage and sclerosis in the background (Fig. 4A). Microscopically, the alveoli in the necrotic lesion were filled with necrotic exudate, nuclear debris and neutrophils. There were scattered virus-infected cells with intranuclear inclusion bodies of Cowdry type A, full and smudge types, and the inclusion bodies in and around the necrotic lesion were immunoreactive for adenovirus (Fig. 4B, C). Gram, PAS and Grocott staining did not detect any bacteria or fungi in the lungs. Findings compatible with pleuritis of the right pleural cavity and peritonitis under the diaphragm with gelatinous fibrin exudate were noted. There were several adenovirusinfected cells in these lesions and in the liver and adrenal glands. Taken together, the patient was diagnosed with a disseminated adenovirus infection.

\section{Discussion}

In hematopoietic stem cell transplant (HSCT) recipients, adenovirus can be detected in the stool, secretions from the nose and throat and sputum two to 150 days posttransplantation (3). Children are at greater risk of developing adenovirus infection than adults $(3,4)$. Other risk factors include treatment with alemtuzumab (5), T cell depletion in grafts $(6)$, acute $\operatorname{GVHD}(7,8)$ and severe lymphocytopenia (less than $0.3 \times 10^{9} / \mathrm{L}$ ) at the time of first detection (6). Disseminated adenovirus disease is defined as the involvement of two or more organs $(9,10)$.

The first patient developed pneumonia after suffering from hemorrhagic cystitis during the early post-transplant period. Adenovirus was detected in the patient's urine only 11 days prior to the development of pneumonia. The detection of adenovirus in the urine rarely indicates disseminated infection (11). We did not treat the first patient with antiviral agents because his hematuria remitted, and no adenovirus was detected in his blood. Although there were no clinical signs of acute GVHD when the patient developed pneumonia, his lymphocyte count was less than $0.5 \times 10^{9} / \mathrm{L}$.

The second patient developed pneumonia during the treatment of chronic GVHD one year after undergoing stem cell transplantation. Lymphocytopenia persisted due to steroid and cyclosporine treatment. She did not have any respiratory symptoms when the lung nodule was detected on CT.

Pulmonary nodules and nodular infiltrates, such as those found in the present two cases, may be caused by fungi and bacteria in HSCT recipients undergoing active immunosuppressive therapy (12). The halos of ground-glass attenuation 

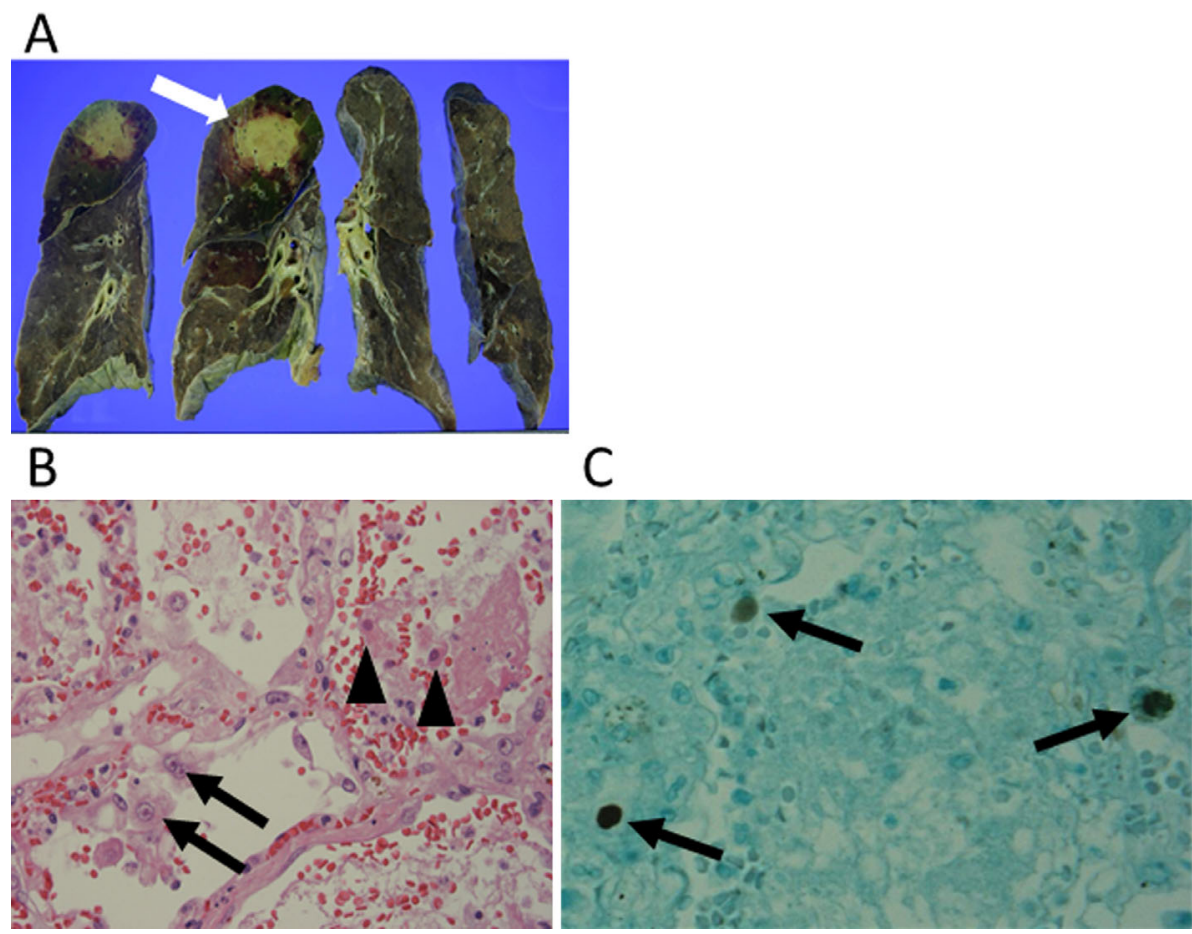

Figure 4. Autopsy findings in Case 2. A: A cut surface shows a 40×30-mm necrotic lesion in the upper lobe of the right lung (arrow). B: A Hematoxylin and Eosin stained lung specimen showing exudate with a few neutrophils and nuclear debris filling the alveolar space. The presence of virus-infected cells containing intranuclear inclusion bodies suggests adenovirus infection $(\times 200)$. Arrows: Cowdry type A inclusion bodies, Arrowheads: smudge cells. C: The intranuclear inclusion bodies exhibit immunoreactivity for adenovirus (arrows). Immunostaining for adenovirus and methyl green $(\times 400)$.

surrounding the nodules observed in our cases are often seen in patients with invasive pulmonary aspergillosis (IPA). The distinguishing shadow is formed by a central necrotic area surrounded by hemorrhage or hemorrhagic infarction due to the proliferation of Aspergillus organisms that spread transbronchially to adjacent pulmonary arterioles (13).

The similarity in the CT and histopathological findings observed between our cases and IPA suggests that adenovirus infections in severely immunocompromised hosts can cause vascular invasion, leading to the formation of nodular shadows with the halo sign.

The mortality rate of all forms of adenovirus infection in HSCT recipients is $26 \%$, while adenovirus pneumonia and disseminated disease tend to show a worse outcome with rates ranging from $50 \%$ to $80 \%$ (1). Ribavirin (14-17) and cidofovir $(2,18-23)$ are used to treat adenoviral infections, with cidofovir having been found to be the most effective thus far (2). However, even cidofovir has limited efficacy once adenovirus disease develops. The efficacy of quantitative viral load measurement followed by the administration of preemptive cidofovir therapy has been reported $(19-22,24)$. Although most of these reports were small retrospective studies, preemptive cidofovir therapy is associated with clinical improvements leading to increased survival (2).

Lymphocyte recovery is crucial for the prevention of or recovery from adenoviral disease $(25,26)$. The adoptive transfer of adenovirus-specific $\mathrm{T}$ cells to pediatric HSCT recipients with adenovirus infection may reduce the viral load and improve the patient's symptoms $(27,28)$. The early detection of adenovirus in the peripheral blood or on organ biopsies and the development of new drugs are crucial for the management of adenovirus infection in HSCT recipients.

The authors state that they have no Conflict of Interest (COI).

\section{References}

1. Ison MG. Adenovirus infections in transplant recipients. Clin Infect Dis 43: 331-339, 2006.

2. Neofytos D, Ojha A, Mookerjee B, et al. Treatment of adenovirus disease in stem cell transplant recipients with cidofovir. Biol Blood Marrow Transplant 13: 74-81, 2007.

3. Baldwin A, Kingman H, Darville M, et al. Outcome and clinical course of 100 patients with adenovirus infection following bone marrow transplantation. Bone Marrow Transplant 26: 1333-1338, 2000.

4. Muller WJ, Levin MJ, Shin YK, et al. Clinical and in vitro evaluation of cidofovir for treatment of adenovirus infection in pediatric hematopoietic stem cell transplant recipients. Clin Infect Dis 41: 1812-1816, 2005.

5. Myers GD, Krance RA, Weiss $H$, et al. Adenovirus infection rates in pediatric recipients of alternate donor allogeneic bone marrow transplants receiving either antithymocyte globulin (ATG) or alem- 
tuzumab (Campath). Bone Marrow Transplant 36: 1001-1008, 2005.

6. Chakrabarti S, Mautner V, Osman H, et al. Adenovirus infections following allogeneic stem cell transplantation: incidence and outcome in relation to graft manipulation, immunosuppression, and immune recovery. Blood 100: 1619-1627, 2002.

7. Flomenberg P, Babbitt J, Drobyski WR, et al. Increasing incidence of adenovirus disease in bone marrow transplant recipients. J Infect Dis 169: 775-781, 1994.

8. Runde V, Ross $S$, Trenschel R, et al. Adenoviral infection after allogeneic stem cell transplantation (SCT): report on 130 patients from a single SCT unit involved in a prospective multi center surveillance study. Bone Marrow Transplant 28: 51-57, 2001.

9. Munoz FM, Piedra PA, Demmler GJ. Disseminated adenovirus disease in immunocompromised and immunocompetent children. Clin Infect Dis 27: 1194-1200, 1998.

10. Chakrabarti S, Donald WM, Moss PAH, et al. Adenovirus infections in stem cell transplant recipients: recent developments in understanding of pathogenesis, diagnosis and management. Leuk Lymphoma 45: 873-885, 2004.

11. Lion $T$, Baumgartinger $R$, Watzinger $F$, et al. Molecular monitoring of adenovirus in peripheral blood after allogeneic bone marrow transplantation permits early diagnosis of disseminated disease. Blood 102: 1114-1120, 2003.

12. Wingard JR, Hiemenz JW, Jantz MA. How I manage pulmonary nodular lesions and nodular infiltrates in patients with hematologic malignancies or undergoing hematopoietic cell transplantation. Blood 120: 1791-1800, 2012.

13. Kuhlman JE, Fishman EK, Siegelman SS. Invasive pulmonary aspergillosis in acute leukemia: characteristic findings on CT, the CT halo sign, and the role of CT in early diagnosis. Radiology 157: 611-614, 1985.

14. Cassano WF. Intravenous ribavirin therapy for adenovirus cystitis after allogeneic bone marrow transplantation. Bone Marrow Transplant 7: 247-248, 1991.

15. Murphy GF, Wood DP Jr, McRoberts JW, et al. Adenovirusassociated hemorrhagic cystitis treated with intravenous ribavirin. J Urol 149: 565-566, 1993.

16. Jurado M, Navarro JM, Hernández J, et al. Adenovirus-associated haemorrhagic cystitis after bone marrow transplantation successfully treated with intravenous ribavirin. Bone Marrow Transplant 15: 651-652, 1995.

17. Miyamura K, Hamaguchi M, Taji H, et al. Successful ribavirin therapy for severe adenovirus hemorrhagic cystitis after allogeneic marrow transplant from close HLA donors rather than distant donors. Bone Marrow Transplant 25: 545-548, 2000.

18. Hoffman JA, Shah AJ, Ross LA, et al. Adenoviral infections and a prospective trial of cidofovir in pediatric hematopoietic stem cell transplantation. Biol Blood Marrow Transplant 7: 388-394, 2001.

19. Bhadri VA, Lee-Horn L, Shaw PJ. Safety and tolerability of cidofovir in high-risk pediatric patients. Transpl Infect Dis 11: 373379, 2009.

20. Ljungman $P$, Ribaud $P$, Eyrich $M$, et al. Cidofovir for adenovirus infections after allogeneic hematopoietic stem cell transplantation: a survey by the Infectious Diseases Working Party of the European Group for Blood and Marrow Transplantation. Bone Marrow Transplant 31: 481-486, 2003.

21. Anderson EJ, Guzman-Cottrill JA, Kletzel M, et al. High-risk adenovirus-infected pediatric allogeneic hematopoietic progenitor cell transplant recipients and preemptive cidofovir therapy. Pediatr Transplant 12: 219-227, 2008.

22. Yusuf U, Hale GA, Carr J, et al. Cidofovir for the treatment of adenoviral infection in pediatric hematopoietic stem cell transplant patients. Transplantation 81: 1398-1404, 2006.

23. Nagafuji K, Aoki K, Henzan H, et al. Cidofovir for treating adenoviral hemorrhagic cystitis in hematopoietic stem cell transplant recipients. Bone Marrow Transplant 34: 909-914, 2004.

24. Platzbecker U, Bandt D, Thiede C, et al. Successful preemptive cidofovir treatment for CMV antigenemia after dose-reduced conditioning and allogeneic blood stem cell transplantation. Transplantation 71: 880-885, 2001.

25. Heemskerk B, Lankester AC, van Vreeswijk T, et al. Immune reconstitution and clearance of human adenovirus viremia in pediatric stem-cell recipients. J Infect Dis 191: 520-530, 2005.

26. Kalpoe JS, van der Heiden PLJ, Barge RMY, et al. Assessment of disseminated adenovirus infections using quantitative plasma PCR in adult allogeneic stem cell transplant recipients receiving reduced intensity or myeloablative conditioning. Eur J Haematol 78: 314-321, 2007.

27. Feuchtinger T, Matthes-Martin S, Richard C, et al. Safe adoptive transfer of virus-specific T-cell immunity for the treatment of systemic adenovirus infection after allogeneic stem cell transplantation. Br J Haematol 134: 64-76, 2006.

28. Leen AM, Christin A, Myers GD, et al. Cytotoxic T lymphocyte therapy with donor $\mathrm{T}$ cells prevents and treats adenovirus and Epstein-Barr virus infections after haploidentical and matched unrelated stem cell transplantation. Blood 114: 4283-4292, 2009.

(C) 2014 The Japanese Society of Internal Medicine http://www.naika.or.jp/imonline/index.html 